Rochester Institute of Technology

RIT Scholar Works

5-13-2019

\title{
Approximating Large Scale Arbitrary Unitaries with Integrated Multimode Interferometers
}

\author{
Matthew Van Niekerk \\ Rochester Institute of Technology \\ Jeffrey A. Steidle \\ Rochester Institute of Technology \\ Gregory A. Howland \\ Rochester Institute of Technology \\ Michael L. Fanto \\ Rochester Institute of Technology
}

Follow this and additional works at: https://scholarworks.rit.edu/other

\section{Recommended Citation}

Matthew van Niekerk, Jeffrey A. Steidle, Gregory A. Howland, Michael L. Fanto, Nicholas Soures, Fatima Tuz Zohora, Dhireesha Kudithipudi, Stefan F. Preble, "Approximating large scale arbitrary unitaries with integrated multimode interferometers," Proc. SPIE 10984, Quantum Information Science, Sensing, and Computation XI, 109840J (13 May 2019); doi: 10.1117/12.2523581

This Conference Proceeding is brought to you for free and open access by the Faculty \& Staff Scholarship at RIT Scholar Works. It has been accepted for inclusion in Presentations and other scholarship by an authorized administrator of RIT Scholar Works. For more information, please contact ritscholarworks@rit.edu. 


\section{Approximating large scale arbitrary unitaries with integrated multimode interferometers}

van Niekerk, Matthew, Steidle, Jeffrey, Howland, Gregory, Fanto, Michael, Soures, Nicholas, et al.

Matthew van Niekerk, Jeffrey A. Steidle, Gregory A. Howland, Michael L. Fanto, Nicholas Soures, Fatima Tuz Zohora, Dhireesha Kudithipudi, Stefan F. Preble, "Approximating large scale arbitrary unitaries with integrated multimode interferometers," Proc. SPIE 10984, Quantum Information Science, Sensing, and Computation XI, 109840J (13 May 2019); doi: $10.1117 / 12.2523581$

Event: SPIE Defense + Commercial Sensing, 2019, Baltimore, Maryland, United States 


\title{
Approximating Large Scale Arbitrary Unitaries with Integrated Multimode Interferometers
}

\author{
Matthew van Niekerk ${ }^{1, *}$, Jeffrey A. Steidle ${ }^{1}$, Gregory A. Howland ${ }^{1}$, Michael L. Fanto ${ }^{1,2}$, \\ Nicholas Soures $^{3}$, Fatima Tuz Zohora ${ }^{3}$, Dhireesha Kudithipudi ${ }^{3}$, and Stefan F. Preble ${ }^{1}$ \\ ${ }^{1}$ Rochester Institute of Technology, RIT Integrated Photonics, Rochester, NY 14623 USA \\ ${ }^{2}$ Air Force Research Laboratory, Information Directorate, Rome, NY 13441 USA \\ ${ }^{3}$ Rochester Institute of Technology, Neuromorphic AI Lab , Rochester, NY 14623 USA
}

\begin{abstract}
Unitary operations using linear optics have many applications within the quantum and neuromorphic space. In silicon photonics, using networks of simple beam splitters and phase shifters have proven sufficient to realize large-scale arbitrary unitaries. While this technique has shown success with high fidelity, the grid physically scales with an upper bound of $O\left(n^{2}\right)$. Consequently, we propose to considerably reduce the footprint by using multimode interference (MMI) devices. In this paper, we investigate the active control of these MMIs and their suitability for approximating traditionally used unitary circuits.
\end{abstract}

Keywords: Unitary, Linear Operator, Multimode Interferometer, Silicon Photonics, Neuromorphic Computing, Quantum Computing

\section{INTRODUCTION}

Silicon photonics has become a leading candidate for both quantum and neuromorphic computing. Not surprising, there are shared heuristics and architectures in what silicon photonics offers to each pursuit. ${ }^{1-4}$ The overlap lays within the concept of the linear operator, where in quantum computing it acts as the logic gate and in neuromorphic computing it acts as the weight and summation (synapse/axon). As far as the linear operator is concerned, there are many ways to implement this in an optical domain including interferometers and spectral filters. ${ }^{5}$ The primary approach, however, has become to employ the Reck and Zielinger decomposition method for the Mach-Zehnder Interferometer (MZI) grid or similar variations. ${ }^{1,6-8}$ Making use of the MZI grid has many advantages, most importantly near-full control of the linear operator. However, there is one large roadblock with the design implementation: the physical size limit of the grid grows at complexity $O\left(n^{2}\right) .{ }^{9}$ Some of the variations on the decomposition ${ }^{7,8}$ have shown that the limit can be as low as $O(n \log (n))$. We were interested by the challenge of finding an innovative way to solve this problem, so we decided to revisit the multimode interferometer (MMI) for a novel approach.

In this work we propose an alternative approach that uses an actively tuned MMI to realize near-arbitrary tuning of unitary operations in a compact footprint. The MMI has traditionally been used as a coupling or splitting device, by leveraging the self-imaging effect. ${ }^{10}$ Since the MMIs self-imaging and propagation behavior is naturally dependent on the material's refractive index, there is an opportunity to actively control the propagation of light through thermo-optic or electro-optic effects. Our methodology in this work revolves around designing, simulating and fabricating devices that enable localized index perturbation in an MMI to realize a condensed alternative to the MZI grid. With the new device, we envision many applications including a compact component for neuromorphic photonic computing.

\footnotetext{
*M.v.N: E-mail: mv7146@rit.edu
} 


\section{DEVICE DESIGN AND FABRICATION}
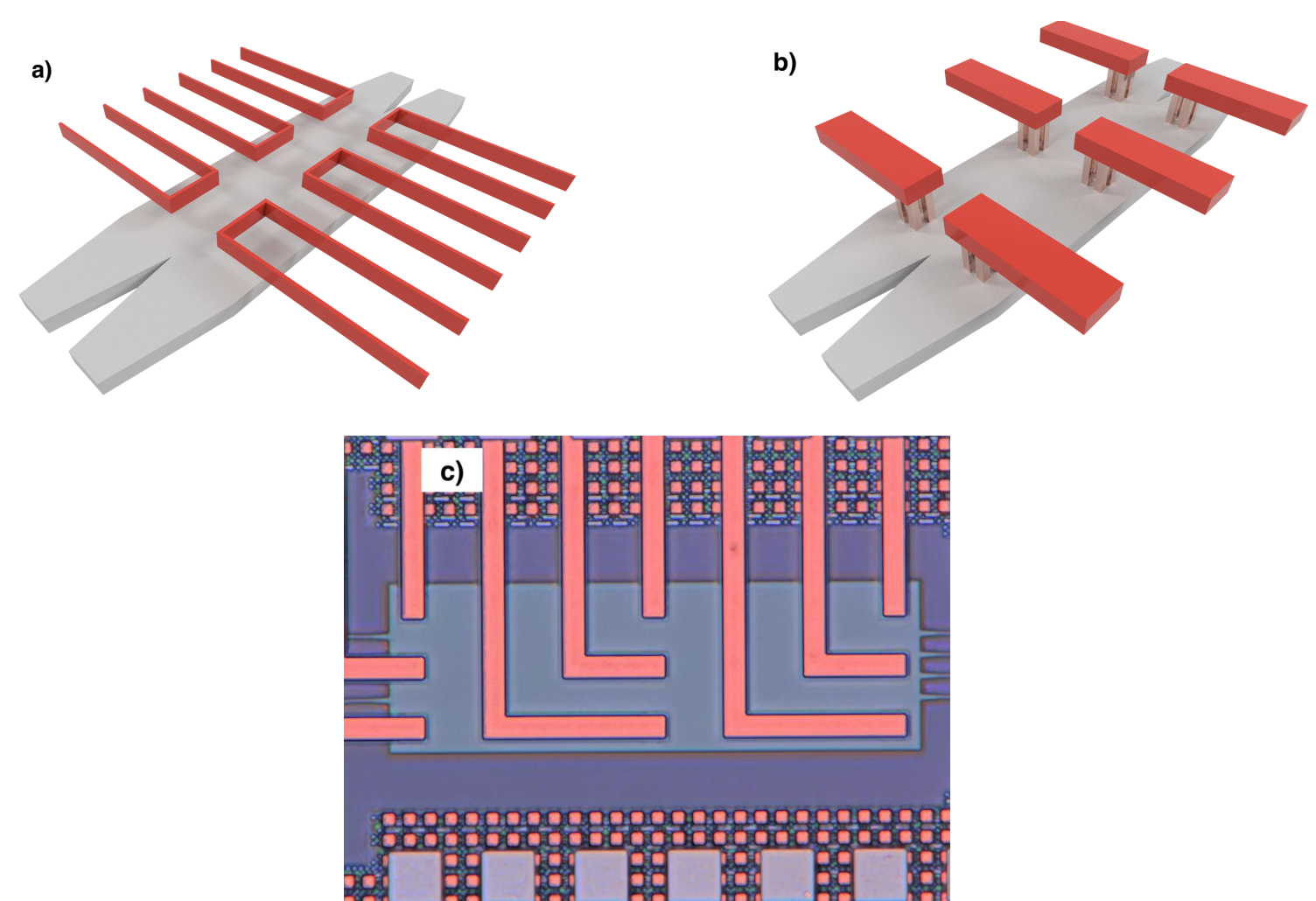

Figure 1: (a) The thermo-optic approach, where we introduce thin metal wires as resistors some distance above the MMI slab, so as to introduce a heat-induced index profile. (b) The electro-optic approach, where we introduce direct contact of the metal vias into the MMI slab. The slab was doped with low concentration, and where the metal via contacts are present a high concentration was introduced. The contacts will act together as siliconresistors, which can have both electro-optic and thermo-optic effects within the slab. (c) A 3000X microscope image of the recently fabricated electro-optic approach over a 4x4 MMI with 9 metal via contacts.

In this initial phase of the development of our idea, we designed some different schemes for creating index control of the MMI slab. Creating a device that allows for active large scale tuning of the material index with thermooptic or electro-optic effects in a localized area is a near impossibility in a standard fabrication process line. With this in mind, we designed two permutations of an active MMI, one for thermo-optic and one for electro-optic, shown in Figure 1.

Figure 1 (a) shows an approach for the thermo-optic active MMI. The idea is to densely pack thin metal wires to act as "local" heat induced index perturbators. The challenge here is to find ways to pack the wires densely enough while accounting for the electrical interconnect bottleneck. Figure 1 (b) shows our electro-optic approach, which is designed to directly contact the doped silicon and create in-place resistors to aid in beam steering. The challenge with this design is to determine the acceptable loss introduced into the system with the metal vias and the doped regions. This solution most likely will struggle in quantum experiments (dealing with single photons), but should still have high enough throughput for the neural network circuit demands. Figure 1 (c) is a microscope image of our recently fabricated electro-optic MMI, while we are still waiting for the thermo-optic MMI to be completed. Our design process involved many simulations of particular passive MMI devices. However, for our active designs, simulations are less appropriate due to the high density of materials and doping. In light of this, our approach changed from multi-physics simulations to analyzing index perturbation and inverse design. 


\section{ACTIVE CONTROL OF AN MMI}

The large-scale, arbitrary unitary MZI grid is an $N$ by $N$ grid where each MZI can be tuned to realize any linear transformation. ${ }^{8}$ Figure 2 shows the schematic representation of the MZI grid, where each crossing represents an MZI and a phase shifter. The key of the decomposition is that the product of the matrices is physically represented by the components in the grid. ${ }^{6}$ Our goal is to approximate such a circuit design with a single tunable $N$ by $N$ port MMI, in turn significantly reducing footprint. In contrast to the MZI grid, an MMI's transfer matrix is traditionally unchanging. However, in Figure 3 we show our concept with an varying index control function. Figure 3 showcases the stark difference between the MZI and the MMI concept; the MZI requires a factoring of multiple unit cells whereas the MMI is both the unit cell and the complete linear operator circuit.

a)

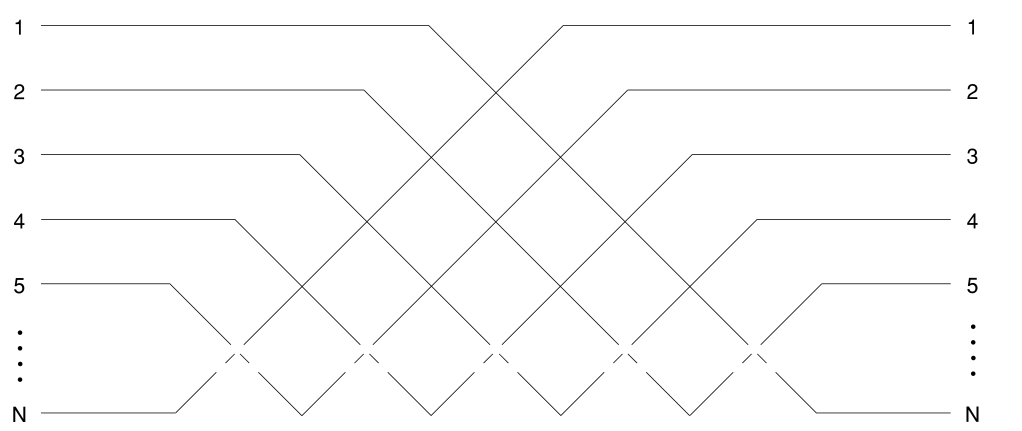

b)

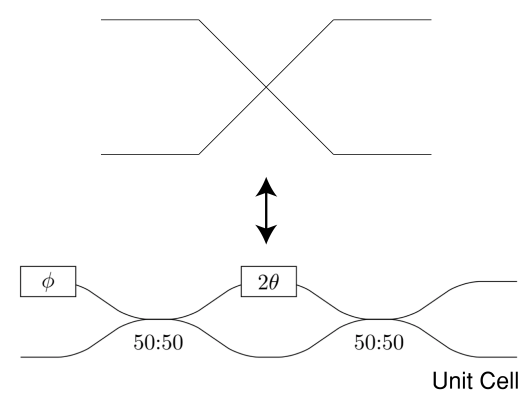

Figure 2: a) A general representation of the MZI grid using the Reck decomposition, ${ }^{6,8}$ where between 5 and $N$ it would follow a similar construction pattern to arrive at the circuit design. b) The crossings in the main architecture represent the unit cell, which is an MZI with an extra phase shifter. In the unit cell the boxes indicate the phase shifters, $\phi, 2 \theta$ and the 50:50 indicates the beam splitter.

The MMI is difficult to model with increasing $N$, where differing models have direct implications on energy conservation and other complexities. ${ }^{11}$ In Figure 3 we show the MMI with the varying index control function that is controlled by external voltages (where the subscript $m$ depends on the experiment). With complete index control, we could make the light "turn in circles" so to speak, but this is an over-exaggeration of full control in a device like this. Instead, we are interested here in a realistic perspective of the control we can expect with the material limitations we have (i.e. thermo-optic coefficient) without melting our chip. Controlling portions of this varying index control function is analogous to the tuning that exists within a grid of MZI's and as a result must be controlled in order realize arbitrary unitary operation with a MMI. In recent work, Bruck et. al has shown that the MMI can be controlled in this way but the approach was not integrated, did not realize arbitrary control and changed the material permanently. ${ }^{12}$

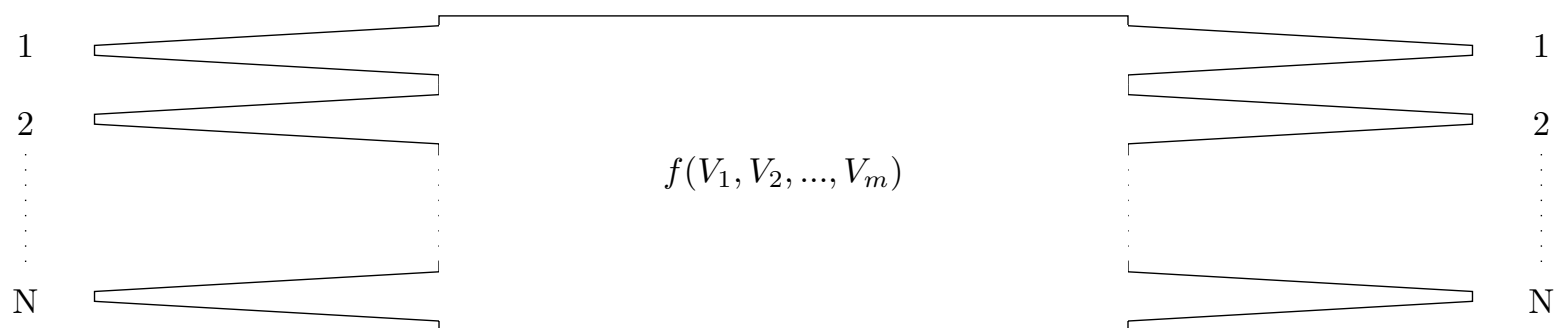

Figure 3: An "active" MMI $N$ by $N$ general component, showing that the MMI can serve as a general $N$ by $N$ operator as a function of the external controls $\left(V_{1}, V_{2}, \ldots V_{m}\right)$. The MMI is intended as both the unit cell and the complete linear operator. 
As mentioned, solving a general equation for any $N$ by $N$ MMI has complicated implications. ${ }^{11}$ Cooney and Peters developed an analytical approach for understanding a general passive MMI transfer function. ${ }^{13}$ Their approach allows for specific 1D and 2D solutions of constant index MMIs, since we can assume (most of) the modes exist completely within the bulk of the material (silicon). In our case, we have to deviate by creating nonconstant or perturbed index throughout the slab of the MMI. In order to develop this solution we decided two different methods: create index perturbation maps inside of our electromagnetic solver and create the solution with inverse design. As shown in Section 2, we needed to keep our simulations tethered to the physical devices we had designed in addition to the realistic material characteristics.

\subsection{Index Perturbation}
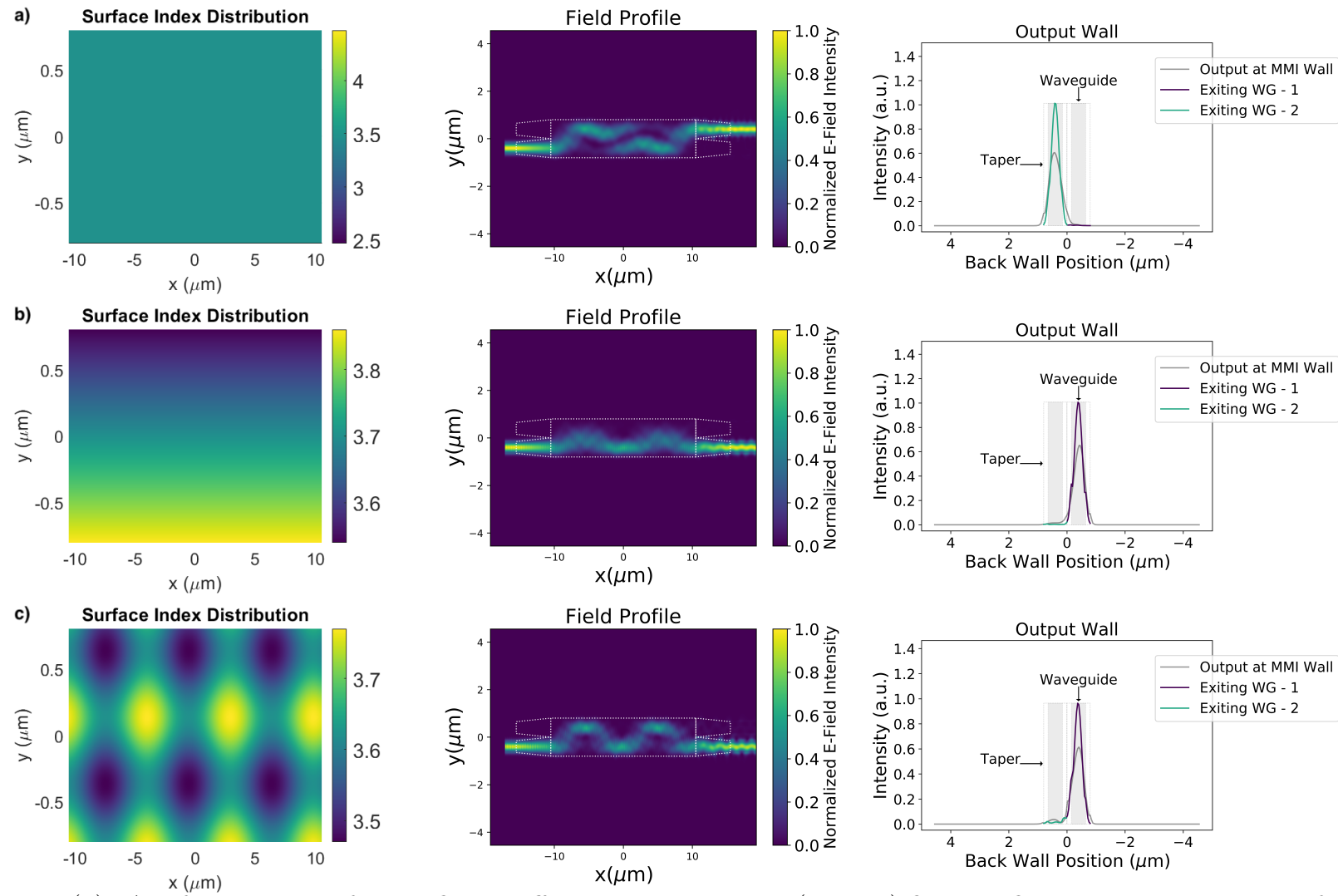

Figure 4: (a): A reference point for the finite difference time domain (FDTD) field profile and output intensity for a non-perturbed MMI. (b): A slightly perturbed surface with higher index toward the bottom in the $y$ direction, acting as an in-place waveguide. (c): A periodically perturbed surface which emulates local hot-spots of index change. Both the (b) and (c) show a complete switch of light guidance from the top output port to the bottom.

We simulated arbitrary index perturbation maps applied to our proposed device to control the index perturbation and realize light steering behavior. These simulations were done using an FDTD solver. Figure 4 shows a proofof-principle example of how the index perturbation can control the coupling of the light through the MMI. In Figure 4 (a) there is no index change throughout the surface. This particular device was designed to move the light from one waveguide diagonally across the MMI. Figure $4(\mathrm{~b}, \mathrm{c})$ show a complete switch of the port through which the light is exiting the structure, demonstrating two ideas for realizing a tunable MMI. Figure 4 (b) shows a perturbed surface that leans to the $-y$ side of the device. Figure 4 (c) similarly shows a periodic perturbation map that also favors the $-y$ side. This tuning scheme demonstrates how a simple perturbation can have a substantial effect over a short distance. We aim to converge on an index map that allows us to have control analogous to the cascading MZI grid. Hence, in the Figure 4 (c) we chose to look at point perturbation (with smoothing) as opposed to the line perturbation in Figure 4 (b). 


\subsection{Inverse Design}

A different, yet powerful, approach is to use inverse design to create the index perturbation for our MMI devices. In recent work, Hughes et. al designed an adjoint and gradient solution for novel nonlinear photonic devices. ${ }^{14}$ Along with their work, they published an open source project with a Finite-Difference-FrequencyDomain (FDFD) solver and inverse optimization solver called angler. ${ }^{14}$ We realized that our architecture 2 applies nearly directly to the method in angler. Whereas the goal in angler is to create novel nonlinear devices, we took advantage of the linear solver. Our fabricated devices will not have point control, as mentioned, so we set the pixel size of our of permittivity topology to a large size. The result is shown in Figure 5.

Figure 5 shows off the angler inverse design process. The user sets the target goal for the device, or the objective function, and the optimization will then continue to edit the permittivity of the shape until it reaches that state where the objective function is satisfied. ${ }^{14}$ However, instead of viewing the final outcome as the device to be fabricated, we are viewing this case as the index perturbation map of the device already fabricated. Figure 5 (a) shows the passive 1x3 MMI device and the FDFD field profile. In Figure 5 (b) the bottom port of the device was targeted, and the permittivity profile was optimized in order to guide the light into that port. In this example, the bottom port saw approximately $97 \%$ of the incident power through the port, with under $1 \%$ in the other two ports. Figure 5 (c) shows a different optimization of the same device, where the goal was to see transmission through the middle port. Again, the permittivity map was optimized to fit the goal. Here the middle port saw over $98 \%$ of the transmission. Future contribution and improvement to angler, if used in this way, would need to focus on incorporating more realistic and experimental material choices. However, we feel that this approach can provide us with a starting point for index perturbation maps in our physical devices.
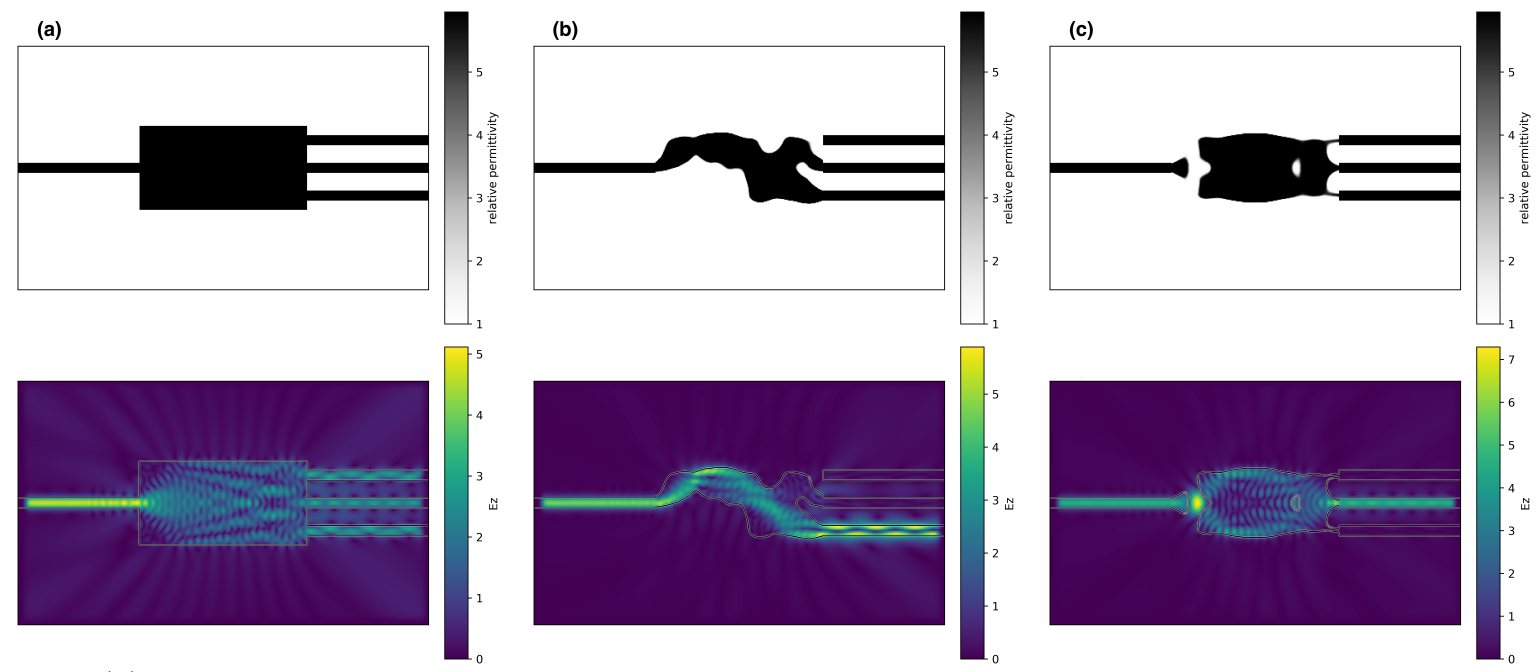

Figure 5: (a) The top figure is the permittivity distribution of the starting example 1x3 passive MMI shape. Below is the FDFD field profile of the MMI. (b) The top figure is the permittivity distribution of the 1x3 MMI targeted at the bottom port. Below is the FDFD field profile showing transmission through to the bottom port. (c) The top figure is the permittivity distribution of the $1 \times 3$ MMI targeted at the middle port. Below is the FDFD field profile showing transmission through the middle port. All of these plots were generated with the angler software. ${ }^{14}$

\section{APPLICATIONS}

For this project, we feel that the application space is quite large. We envision the active MMI as a device which can serve in multiple neural network topologies. Preliminary simulation work by our group has shown that a re-configurable MMI can compete with state-of-the-art networks. ${ }^{15}$ Experimental approaches are currently underway to investigate these results among other benchmarks. Additionally, our group has a long reaching goal of testing the thermo-optic MMI device in quantum experiments. The MMI is a good candidate for certain 
tasks within quantum photonics ${ }^{11}$ however realizing a full linear circuit approximation with high fidelity from a silicon foundry is a more distant goal.

\section{CONCLUSION}

In the initial phase of this project, our group has designed two unique approaches to create an active MMI that can approximate the traditional MZI grid. The thermo-optic and electro-optic approaches are each unique in their ability to allow for beam steering and index perturbation within the MMI slab. We used FDTD simulations to impose different, approximate index perturbation schemes onto the MMI. We also investigated inverse design with angler ${ }^{14}$ to instead produce the index maps for particular configurations of desired outputs. Our devices have recently been fabricated and experimental investigation is underway.

\section{ACKNOWLEDGMENTS}

This material is based upon work supported by the National Science Foundation under Grant No. ECCS-1810282 and by AIM Photonics and sponsored by Air Force Research Laboratory via agreement number FA8650-15-25220. The U.S. Government is authorized to reproduce and distribute reprints for Governmental purposes notwithstanding any copyright notation thereon. The views, opinions, conclusions, and findings contained herein are those of the authors and should not be interpreted as necessarily representing the official policies or endorsements, either expressed or implied, of National Science Foundation, Air Force Research Laboratory, or the U.S. Government. The authors would like to thank contributions of Air Force Research Laboratory's quantum information science group.

\section{REFERENCES}

[1] Shen, Y., Harris, N. C., Skirlo, S., Prabhu, M., Baehr-Jones, T., Hochberg, M., Sun, X., Zhao, S., Larochelle, H., Englund, D., et al., "Deep learning with coherent nanophotonic circuits," Nature Photonics 11(7), 441 (2017).

[2] Hamerly, R., Sludds, A., Bernstein, L., Soljačić, M., and Englund, D., "Large-scale optical neural networks based on photoelectric multiplication," arXiv preprint arXiv:1812.07614 (2018).

[3] Steinbrecher, G. R., Olson, J. P., Englund, D., and Carolan, J., "Quantum optical neural networks," arXiv preprint arXiv:1808.10047 (2018).

[4] Harris, N. C., Carolan, J., Bunandar, D., Prabhu, M., Hochberg, M., Baehr-Jones, T., Fanto, M. L., Smith, A. M., Tison, C. C., Alsing, P. M., et al., "Linear programmable nanophotonic processors," Optica 5(12), 1623-1631 (2018).

[5] Peng, H.-T., Nahmias, M. A., de Lima, T. F., Tait, A. N., and Shastri, B. J., "Neuromorphic photonic integrated circuits," IEEE Journal of Selected Topics in Quantum Electronics 24(6), 1-15 (2018).

[6] Reck, M., Zeilinger, A., Bernstein, H. J., and Bertani, P., "Experimental realization of any discrete unitary operator," Physical review letters 73(1), 58 (1994).

[7] Jing, L., Shen, Y., Dubek, T., Peurifoy, J., Skirlo, S., LeCun, Y., Tegmark, M., and Soljai, M., "Tunable efficient unitary neural networks (eunn) and their application to rnns," (2016).

[8] Clements, W. R., Humphreys, P. C., Metcalf, B. J., Kolthammer, W. S., and Walmsley, I. A., "Optimal design for universal multiport interferometers," Optica 3(12), 1460-1465 (2016).

[9] Aaronson, S. and Arkhipov, A., "The computational complexity of linear optics," in [Proceedings of the forty-third annual ACM symposium on Theory of computing], 333-342, ACM (2011).

[10] Wen, J., Zhang, Y., and Xiao, M., "The talbot effect: recent advances in classical optics, nonlinear optics, and quantum optics," Adv. Opt. Photon. 5, 83-130 (Mar 2013).

[11] Peruzzo, A., Laing, A., Politi, A., Rudolph, T., and O'Brien, J. L., "Multimode quantum interference of photons in multiport integrated devices," Nature Communications 2, 224 EP - (Mar 2011).

[12] Bruck, R., Vynck, K., Lalanne, P., Mills, B., Thomson, D. J., Mashanovich, G. Z., Reed, G. T., and Muskens, O. L., "All-optical spatial light modulator for reconfigurable silicon photonic circuits," Optica 3, 396-402 (Apr 2016). 
[13] Cooney, K. and Peters, F. H., "Analysis of multimode interferometers," Optics express 24(20), 22481-22515 (2016).

[14] Hughes, T. W., Minkov, M., Williamson, I. A. D., and Fan, S., "Adjoint method and inverse design for nonlinear nanophotonic devices," ACS Photonics 5(12), 4781-4787 (2018).

[15] Soures, N., Steidle, J., Preble, S., and Kudithipudi, D., "Neuro-mmi: A hybrid photonic-electronic machine learning platform," in [2018 IEEE Photonics Society Summer Topical Meeting Series (SUM)], 187-188, IEEE (2018). 